genital tract disease, screening for this bacterium should be considered, particularly with a surgical procedure such as termination of pregnancy, although the lack of a commercial test is problematic.

\section{3-S6.03 THE DIAGNOSIS OF LYMPHOGRANULOMA VENEREUM AT ONE'S FINGERTIPS}

doi:10.1136/sextrans-2011-050109.135

T Crucitti, H Smet. Institute of Tropical Medicine, Antwerpen, Belgium

Background Outbreaks of Lymphogranuloma venereum (LGV) in sexual networks of men who have sex with men (MSM) are reported in several countries in Europe. Although accurate laboratory diagnosis is required to provide adequate patient management, the laboratory identification of LGV can be problematic.

Objective To establish a fast and reliable testing algorithm for the identification of Chlamydia trachomatis L serovars.

Methods Previously, anal specimens from MSM suspected to be positive for $C$ trachomatis were tested with a testing algorithm using commercial molecular amplification assays. Confirmed $C$ trachomatis samples were then analysed in batches by RFLP to identify the L serovars. From September 2010 onwards, the Abbott CT/NG Real Time PCR has been used for the detection of $C$ trachomatis in biological specimens collected at or referred to the ITM for Chlamydial infection diagnosis. Furthermore, confirmation of $C$ trachomatis and identification of the $L$ serovar types are performed with an in-house Real Time PCR assay. This assay uses DNA extract obtained with the Abbott assay. The selection of the primers and test procedure is based on the publication by Chen et al. and includes two specific probes for the detection of the $\mathrm{L}$ and the non $\mathrm{L}$ serovars.

Results Out of a total of 940 samples tested with the new methods, we detected $58(6.2 \%)$ positive samples for $C$ trachomatis and of those $12(20.7 \%)$ were L serovars. Eight were detected in specimens collected from the anus, two in urethral specimens, one in urine, and one in a vaginal specimen. All non vaginal specimens were collected from men. With the Abbott CT/NG Real Time PCR for screening and the in house RT PCR assay for confirmation, we were able to confirm positive results for $C$ trachomatis and to distinguish the $\mathrm{L}$ serovar from the non $\mathrm{L}$ serovar types within 2 days after specimen reception. In addition the in house RT PCR assay was more sensitive, more discriminative and at least 4 times cheaper compared to the RFLP method.

Conclusion The detection of $\mathrm{L}$ serovar of $C$ trachomatis can be done on a routine basis at a very acceptable cost and test around time. The L serovar types may be more frequent in Belgium then initially thought, they are present in various biological specimens and possibly in women.

\section{Abstract 03-S6.03 Table 1 LGVV Testing}

\begin{tabular}{lcccl}
\hline Year & specimens tested $^{*}$ & L serovar & non L serovar & not typable \\
\hline 2011 January & 196 & 4 & 11 & 0 \\
2010 Sept-Dec & 744 & 8 & 35 & 0 \\
2010 Jan-Aug & 23 & 14 & 9 & 0 \\
2009 & 23 & 17 & 5 & 1 \\
2008 & 13 & $11 \dagger$ & 1 & 1 \\
2007 & 19 & 13 & 5 & 1 \\
2006 & 14 & 7 & 4 & 3 \\
2005 & 6 & 2 & 1 & 3 \\
2004 & 11 & 8 & 2 & 1
\end{tabular}

*Until September 2010 only male anal specimens or rectal biopsies were tested except for 2008 when 2 specimens from penile ulcers were tested.

†Two penile ulcer specimens.

\section{3-S6.04 MULTI-SITE SCREENING FOR LYMPHOGRANULOMA VENEREUM (LGV) IN THE USA}

doi:10.1136/sextrans-2011-050109.136

${ }^{1} \mathrm{~J}$ Hardick, ${ }^{1} \mathrm{~N}$ Quinn, ${ }^{1} \mathrm{~S}$ Eshelman, ${ }^{1} \mathrm{E}$ Piwowar-Manning, ${ }^{1} \mathrm{~V}$ Cummings, ${ }^{2} \mathrm{~V}$ C Marsiglia, ${ }^{1} \mathrm{C}$ A Gaydos, ${ }^{3}$ The HPTN $06{ }^{1}$ Study Team. . ${ }^{1}$ Johns Hopkins University School of Medicine, Baltimore, USA; ${ }^{2}$ Baltimore City Health Department, Baltimore, USA; ${ }^{3} \mathrm{HIV}$ Prevention Trials Network, Washington, USA

Background Lymphogranuloma venereum is a clinical condition caused by infection with one of the Chlamydia trachomatis (CT) L serovars. Proper diagnosis of LGV is critical as the treatment varies significantly from antibiotic therapy utilised for other CT infections. LGV has re-emerged in Europe as an important sexually transmitted infection (STI), particularly in men who have sex with men (MSM), due to an outbreak in the Netherlands. LGV surveillance data for the USA is lacking as LGV screening is not routinely performed, even in high risk populations. This study presents LGV surveillance data from multiple sites in the USA.

Methods 1671 rectal samples from African-American MSM participating in a study of the HIV Prevention Trials Network (HPTN 061) BROTHERS Project, were collected from six different cities Los Angeles and San Francisco CA, Atlanta, GA, Boston MA, Washington D.C., New York, NY; and tested for CT by Aptima Combo 2 (Gen-Probe). Additionally, 127 samples from men from Baltimore, $\mathrm{MD}$ who reported anal sex or were symptomatic for CT, and had rectal swabs positive for CT by Combo 2 were also included. All samples were screened for LGV utilising a previously verified LGV specific real-time PCR to determine if the samples were positive for any one of the CT L serovars.

Results Of the 1671 HPTN 061 samples, 112 (6.75\%) were positive for CT and 102 of these have been screened thus far for LGV; none were LGV +. Of $127 \mathrm{CT}+$ samples from Baltimore, two were LGV+. Thus, of $229 \mathrm{CT}+$ rectal samples, only $2(0.87 \%)$, tested positive for LGV by real-time PCR.

Conclusions Less than 1\% of the CT positive samples obtained from rectal swabs from MSM in the US tested positive for LGV. The samples utilised for this study were from a population presumably at high risk for acquisition of LGV, as all samples tested were from men who had either tested positive for CT, reported anal sex, or were symptomatic for CT infection. The prevalence for LGV in this study was quite low, while the non LGV CT prevalence was high in African American MSM from the six cities. Concomitant STDs are thought to drive the disproportionate HIV epidemic among African American MSM and the low prevalence of LGV in this study is of interest. LGV has re-emerged as an important STI in Europe, however this data suggests that it has either not re-emerged in the U.S. or has re-emerged in a population that is not being screened.

03-S6.05 PERCEPTIONS ON POINT-OF-CARE TESTS FOR SEXUALLY TRANSMITTED INFECTIONS-disconnect between frontline clinicians and professionals in industry

doi:10.1136/sextrans-2011-050109.137

Yu-Hsiang Hsieh, C Gaydos, T Hogan, O Uy, J Jackman, Mary Jett-Goheen, A Rompalo. Johns Hopkins University, Baltimore, USA

Background Some recently developed or available sexually transmitted infection (STI) point-of-care tests (POCTs) are not very accurate and are not feasible for use in clinical settings. We conducted a study to determine if a gap exists between STI clinicians/academic experts and industry professionals regarding perceptions of the ideal types and characteristics of STI POCTs. Methods Our online survey design informed by a large-scale focus group study among STI professionals contained sections on 